\title{
The Effect of Alteration of Vertical Dimension of Occlusion on Brain Activity in Complete Denture Wearers
}

\author{
Ayman A. Elmorsy ${ }^{1 *}{ }^{1 *}$, Mohamed Zaki ${ }^{2}$ D, Hafiz Elbahnaswi ${ }^{2}$, Amani R. Moussa ${ }^{1}$, Asmaa N. Elboraey ${ }^{1}$ (D) \\ ${ }^{1}$ Department of Fixed and Removable Prosthodontics, Oral and Dental Research Division, National Research Centre, Cairo, \\ Egypt; ${ }^{2}$ Department of Removable Prosthodontics, Faculty of Dental Medicine, Al-Azhar University, Cairo, Egypt
}



\section{Abstract}

BACKGROUND: The establishment of appropriate vertical dimension of occlusion (VDO) is paramount for successful and functioning complete dentures (CDs).

AIM: The aim of this study was to assess the effect of alteration of VDO on the brain activity in CD wearers.

MATERIALS AND METHODS: Ten completely edentulous patients participated in this study. Each patient received three sets of CDs: one control set and two duplicates' CDs. The control CDs was designed with appropriate VDO (control - VDO), while the duplicate CDs were designated as followed: one with VDO lowered by $-3 \mathrm{~mm}$ (low - VDO) the other one VDO was increased by $+5 \mathrm{~mm}$ (high - VDO). The effect of alteration of VDO on brain activity was assessed by measuring alpha waves changes using electroencephalogram (EEG) before and after chewing gum. Statistical analysis was carried out using one-way ANOVA and dependent t-test. The significance level was set at $\mathrm{p}<0.05$.

RESULTS: There was a significant increase in the alpha waves mean values after chewing $(p<0.05)$ gum compared to before except for low - VDO CDs $(p>0.05)$. The control-VDO revealed a statistically significant higher mean values compared to the duplicate dentures. CDs with high - VDO showed higher mean value than low - VDO dentures, however, it was statistically insignificant $(p>0.05)$.

CONCLUSION: Appropriate determination of VDO is not only important for the success of CDs but also for the functional augmentation of brain activity.

\section{Introduction}

Complete dentures (CDs) have been the treatment option for completely edentulous individuals for more than one century. It restores the lost dentition, masticatory function, speech, and facial proportion of the individual [1]. Although, implant-retained or supported prosthesis is considered as the ultimate treatment for complete edentulism. CDs are still the treatment of choice owing to their economic and healthrelated aspects [2].

The upgrading of dental materials, processing techniques, and introduction of the application Computer Aided-Design/Computer-Aided-Manufacture (CAD/CAM) have simplified the clinical steps and manufacturing procedures of CDs [3]. The number of visits was reduced to two or three visits instead of five visits in the conventional technique [3], [4]. The first visit includes impression making, determination of vertical dimension of occlusion (VDO) of occlusion, and bite registration. The second visit (optional) is for try-in. The last one is for denture delivery. Despite the impressive advances of CAD/CAM in denture manufacturing; the determination of VDO depends on the clinician management [5]. In literature, there are several techniques described to adjust VDO properly [6]. However, there is a possibility of increasing or decreasing VDO, due to manipulation errors may occur.

Appropriate determination of VDO is a challenging step as it is not only crucial for the success of CDs regarding to patient's esthetic and oral function [5]. But also, several studies have been correlated between the successful removable prosthesis and mastication to certain brain activities [7], [8], [9]. They suggested that tooth loss is associated with an increase in the level of plasma glucocorticoid which attenuates the trigeminal nerve and neurotransmitters. As a result, alteration of central nervous function occurs [10]. Alongside, oral sensory information from the trigeminal nerve is diminished, causing inhibition of the brain activities such as learning and memory [11].

The relation between CDs rehabilitation and brain activity has been investigated in previous studies [12], [13], [14]. However, changes in brain activity in CD wearers with increase or decrease in VDO have not been sufficiently evaluated. Therefore, 
the aim of the present study was to assess the effect of alteration of VDO in CD wearers on brain activity using an electroencephalogram (EEG).

\section{Materials and Methods}

Ten completely edentulous patients were selected from the out-patients' clinic of Al-Azhar University Faculty of Dental Medicine and Medical Centre of excellence at National Research Centre, (NRC) Cairo - Egypt.

\section{Ethical approval}

This study was approved by the Medical Research Ethical Committee of NRC, Cairo-Egypt (MREC Approval No. 15120), which is in accordance with the Helsinki Declaration of 1975. All patients were informed about the practical steps of this study and signed a written approval consent.

\section{Inclusion criteria}

All the participants were fulfilling the following criteria: (1) completely edentulous. (2) absence of any brain diseases (e.g. cerebral infarction and Alzheimer's), (3) no psychiatric illness, and (4) skeletally angle's class I.

\section{Exclusion criteria}

Patients having any of the following criteria were excluded: (1) Temporomandibular joint disorders, (2) parafunctional oral habits, and (3) smoker.

\section{Methods \\ Fabrication of CDs}

Each patient received three sets of CDs: control CDs and two types of duplicate CDs. Control CD had appropriate VDO (control - VDO) while duplicate CDs were designed with altered VDO as followed: VDO was lowered by $-3 \mathrm{~mm}$ (low - VDO) and the other one VDO was increased by $+5 \mathrm{~mm}$ (high - VDO) as reported by Matsuda et al., 2014 [15].

Control CDs were fabricated using heat-cured acrylic resin following the standard procedures. After finishing and polishing of dentures they were delivered to participant and all the necessary adjustment was performed. For each control denture set, three duplicates were made using duplicating silicone impression material (Replisil, N 22, Siliconic, GmbH and Co.KG, Germany). One duplicate of the maxillary denture and two duplicates of mandibular casts. On mandibular casts, the duplicate dentures were fabricated; One denture with decreased VDO by $3 \mathrm{~mm}$ (low - VDO) and the other one with increased VDO by +5 mm (high - VDO) [15].

To ensure that duplicate dentures are identical in all aspects as the control one except for the VDO. A semi-adjustable articulator (Bio-Art Brazil) and facebow transfer (bioart, Brazil) record were used to ensure the exact orientation of the maxillary arch to the skull. To fabricate duplicate dentures the incisal guide pin of the articulator was lowered by $3 \mathrm{~mm}$ for low - VDO dentures. For the other duplicate denture, the incisal guide pin was raised by $5 \mathrm{~mm}$ (high - VDO). Each participant was asked to try in duplicate dentures to make any necessary adjustment and to ensure patient comfort.

\section{Measurement of alpha waves using EEG}

Assessment of alpha waves was performed after 1 month of denture wear to ensure patient's adaptation and adequate denture function. Alpha waves records were accomplished while the participant was seated comfortably at rest using EEG (EB neuro, Galileo software, Italy). Records were obtained using 21-channel paste-less bridge electrodes arranged on the scalp (EB Neuro S.P.A) According to the international 10-20 system with reference electrodes placed on both earlobes. Galileo Suite software separates the alpha waves which occur in frequency range of $8-12 \mathrm{~Hz}$, evaluating its power and amplitude.

EEG was performed 3 min immediately before chewing non-flavored gum and one minute after chewing wearing three types of dentures (control, low - VDO, and high - VDO), i.e., the total number of measurement setting was six for each participant. A 30-min time interval was allowed between measurement setting for each denture type.

\section{Statistical analysis}

Data were presented as mean (M) and standard deviation (SD). Statistical analysis was performed with SPSS 16 ® (Statistical Package for Scientific Studies), Graph pad prism and windows excel. Comparison of alpha waves of control and two duplicate dentures (low - VDO and high - VDO) before and after chewing were performed using One Way ANOVA followed by Tukey`s post-hoc test for multiple comparisons. On the other hand, comparison of alpha wave changes for each denture type before and after chewing was performed using Paired t-test. The significance level ( $p$-value) was set at $p<0.05$. 


\section{Results}

The results of measurements of alpha waves for the control and duplicate dentures were presented in (Table 1 and Figures 1 and 2).

Table 1: Alpha waves changes in the three CDs designs (control, low VDO and high VDO) before and after chewing

\begin{tabular}{|c|c|c|c|c|c|c|c|}
\hline \multirow{3}{*}{$\begin{array}{l}\text { EEG } \\
\text { Measurements }\end{array}$} & \multirow{2}{*}{\multicolumn{2}{|c|}{$\begin{array}{l}\text { Control CDs } \\
\text { Control VDO }\end{array}$}} & \multicolumn{4}{|c|}{ Duplicate CDs } & \multirow[t]{3}{*}{$\mathrm{p}$-value } \\
\hline & & & \multicolumn{2}{|c|}{$\begin{array}{l}\text { Low VDO (-3 } \\
\mathrm{mm})\end{array}$} & \multicolumn{2}{|c|}{$\begin{array}{l}\text { High VDO } \\
(+5 \mathrm{~mm})\end{array}$} & \\
\hline & $\mathrm{M}$ & SD & $\bar{M}$ & SD & $M$ & SD & \\
\hline Before Chewing & $1.2^{\mathrm{a}}$ & 0.4 & $0.8^{\mathrm{ab}}$ & 0.4 & $0.4^{\mathrm{b}}$ & 0.5 & $0.002^{*}$ \\
\hline After Chewing & $1.8^{\mathrm{a}}$ & 0.4 & $0.8^{\mathrm{b}}$ & 0.3 & $0.6^{\mathrm{b}}$ & 0.24 & $0.002^{*}$ \\
\hline $\mathrm{p}$-value & \multicolumn{2}{|l|}{$0.002^{*}$} & \multicolumn{2}{|c|}{$1.00 \mathrm{NS}$} & \multicolumn{2}{|c|}{$0.03^{*}$} & \\
\hline
\end{tabular}

Comparison of alpha wave values before chewing gum revealed that control dentures recorded the highest value followed by low - VDO and least was for high - VDO. And the results were statistically significant $(p<0.05)$.

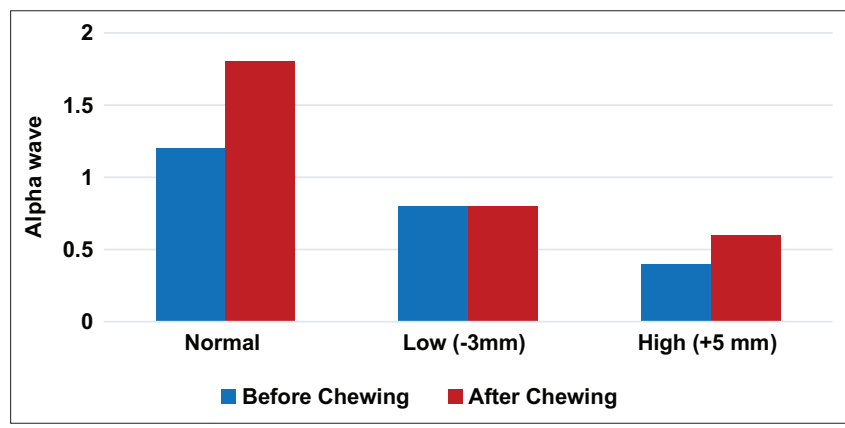

Figure 1: Bar chart represents alpha wave changes of three complete dentures designs (control, low vertical dimension of occlusion (VDO), and high VDO) before and after chewing

After chewing gum: There was an increase in mean values of both control and high - VDO dentures. While low - VDO showed no change in alpha wave. The control recorded the highest value, followed by low - VDO (no change) and high - VDO dentures. Statistical analysis of the three denture sets revealed a significance difference between control and other two denture sets $(p<0.05)$. On the other hand, there was an insignificant difference between low and high - VDO dentures $(p>0.05)$.

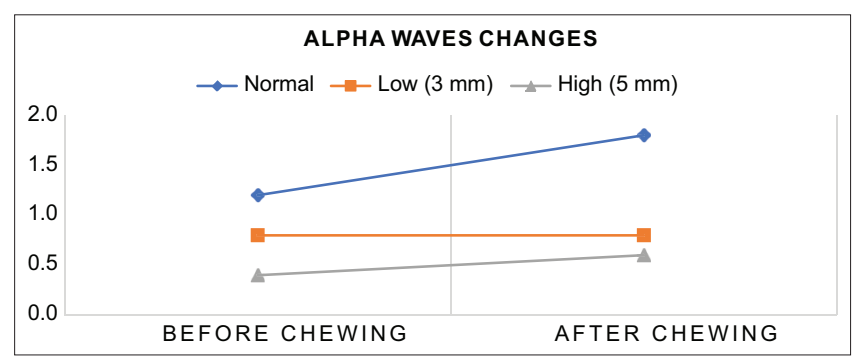

Figure 2: Line chart for mean increase in alpha waves in control and two duplicate dentures before and after chewing

\section{Discussion}

In the present study, the effect of VDO alteration on the brain activity of CD wearers was explored using duplicate dentures of the original functioning one. Duplicate dentures were used to ensure that they were identical to control ones except for VDO changes. For ethical reasons, these dentures were only used during recording EEG tasks. Assessment of brain activity was performed after 1 month of wearing CDs to accomplish all necessary denture adjustments; as well as to guarantee patient adaptation and denture function [16].

In literature, there are several methods reported to assess the brain activity. Among noninvasive, economic, and simplest techniques is EEG. It detects electrical changes in the brain over a period of time. It distinguishes between four types of brain waves; alpha waves were the most relevant to dental research [17], [18]. It reflects the relaxation, anxiety, and stress. Alpha waves value below nine suggests increased risk of dementia [19], [20].

Loss of VDO in dentures wearers may occur due to wear of artificial teeth [21]. On the other hand, increase in VDO could be attributed to manipulation errors either during fabrication of new dentures or relining of the existing one. In both circumstances, the masticatory muscle function and mandibular position are inevitably affected [22], [23].

Banu et al., 2016 [14] mentioned that the occlusion of satisfactory dentures transmits sensory and motor information through receptors present in the periosteum, oral mucosa, and masticatory apparatus to the brain [14], [24]. Therefore, this study aimed to detect the role of VDO and how VDO changes can impact the brain activity.

In the current study, a comparison between three sets of CD: control- with appropriate VDO, and duplicate dentures (low - VDO and high - VDO) was accomplished using EEG recording. It allowed the assessment of brain activity by detecting the alpha wave changes before and after gum chewing.

Determination of appropriate VDO for control dentures was established through combination of metric, phonetic and esthetic methods as well as patient's ability to swallow comfortably without difficulties [23]. On the other hand, the alteration of VDO by decreasing $-3 \mathrm{~mm}$ and increasing $+5 \mathrm{~mm}$ was performed as mentioned by Matsuda et al., [15] This also is advocated by Abduo [25] who demonstrated that permanent increase of VDO up to $5 \mathrm{~mm}$ is acceptable procedure without any harmful effects; and any related signs and symptoms are limited and resolved within 2 weeks.

The results of this comparative study revealed an improvement in alpha waves after chewing gum as 
compared to before chewing expect for the low-VDO dentures. The later demonstrated no changes in alpha waves records after compared to before chewing. Such results are partly supported by previous finding of Hirano et al. 2013 [26], and Perumal et al., 2016 [27]. They reported that chewing was an effective method to stimulate the brain. Perumal et al., 2016 [27] added that restoration of occlusion and masticatory function with satisfactory dentures stimulates the brain activity via trigeminal nerve [27], [15].

Furthermore, the comparative results revealed that the effect of either increasing or decreasing VDO on alpha waves was insignificant. Matsuda et al., 2014 [15] reported a comparable finding in a similar study. However, they stated that there was an insignificant difference between original, high, and low - VDO dentures. They also noticed a significant decrease in the biting force in low - VDO dentures.

Even though, both the control and high - VDO dentures showed an increase in alpha waves. The control dentures presented a significant increase in alpha waves compared to high - VDO. Such outcomes proposed that chewing was not only the key factor for positive enhancement of brain activity, but adequate VDO was a crucial factor. This finding is further supported by the absence of any improvement of brain activity in low - VDO dentures before and after chewing.

The results of the present study were in accordance with Morokuma etal., 2015[20] who concluded that functioning dentures provided better chewing action. This act could augment synaptic/neuronal dysfunction and initiates the stimulation of the central nervous system through the trigeminal nerve. They also documented that satisfactory dentures could be a fundamental factor to improve quality of life and minimize dementia.

\section{Conclusion}

Within the limitations of this pilot study, the following was concluded:

- $\quad$ Appropriate determination of VDO is not only crucial for the success of CDs but also for the functional augmentation of brain activity

- $\quad$ Further clinical studies are required to detect long-term effect of alteration of VDO on brain activity.

\section{Acknowledgments}

The authors would like to thank the Faculty of Dental Medicine, Boys, Cairo, Al-Azhar University for designing and performing the clinical research work; and NRC for the financial support, Dr. Ahmed Mohammed Ezzat Elwan, Research Assis, Department of Children with Special Needs for accomplishing and interpretation of EEG.

\section{References}

1. Wang $W$, Liao $S$, Zhu $Y$, Liu M, Zhao $Q$, Fu $Y$. Recent applications of nanomaterials in prosthodontics. J Nanomater. 2015;2015:408643.

2. Sharka $\mathrm{R}$, Abed $\mathrm{H}$, Hector M. Oral health-related quality of life and satisfaction of edentulous patients using conventional complete dentures and implant-retained overdentures: An umbrella systematic review. Gerodontology. 2019;36(3):195204. https://doi.org/10.1111/ger.12399

PMid:30875108

3. Cunha TR, Della Vecchia MP, Regis RR, Ribeiro AB, Muglia VA, Mestriner $\mathrm{W}$ Jr., et al. A randomised trial on simplified and conventional methods for complete denture fabrication: Masticatory performance and ability. J Dent. 2013;41(2):133-42. https://doi.org/10.1016/j.jdent.2012.09.008

PMid:23000525

4. Neumeier TT, Neumeier H. Digital immediate dentures treatment: Aclinical report of two patients. J Prosthet Dent. 2016;116(3):3149. https://doi.org/10.1016/j.prosdent.2016.01.010 PMid:27061629

5. Calamita M, Coachman C, Sesma N, Kois J. Occlusal vertical dimension: Treatment planning decisions and management considerations. Int J Esthet Dent. 2019;14(2):166-181.

PMid:31061997

6. Discacciati JA, de Souza EL, Vasconcellos WA, Costa SC, Barros VM. Increased vertical dimension of occlusion: Signs, symptoms, diagnosis, treatment and options. J Contemp Dent Pract. 2013;14(1):123-8. https://doi.org/10.5005/ jp-journals-10024-1284 PMid:23579908

7. Ono Y, Yamamoto T, Kubo KY, Onozuka M. Occlusion and brain function: Mastication as a prevention of cognitive dysfunction. J Oral Rehabil. 2010;37(8):624-40. https://doi. org/10.1111/j.1365-2842.2010.02079.x PMid:20236235

8. Okamoto N, Morikawa M, Okamoto K, Habu N, Iwamoto J, Tomioka K, et al. Relationship of tooth loss to mild memory impairment and cognitive impairment: Findings From the Fujiwara-kyo study. Behav Brain Funct. 2010;6:77. https://doi. org/10.1186/1744-9081-6-77

PMid:21194415

9. Krishnamoorthy G, Narayana Al, Balkrishanan D. Mastication as a tool to prevent cognitive dysfunctions. Jpn Dent Sci Rev. 2018;54(4):169-73. https://doi.org/10.1016/j.jdsr.2018.06.001

10. Onozuka M, Watanabe K, Mirbod SM, Ozono S, Nishiyama K, Karasawa $\mathrm{N}$, et al. Reduced mastication stimulates impairment of spatial memory and degeneration of hippocampal neurons in aged SAMP8 mice. Brain Res. 1999;826(1):148-53. https://doi. org/10.1016/s0006-8993(99)01255-x PMid:10216208

11. Terasawa $H$, Hirai $T$, Ninomiya $T$, Ikeda $Y$, Ishijima $T$, Yajima $T$, et al. Influence of tooth-loss and concomitant masticatory alterations on cholinergic neurons in rats: Immunohistochemical and biochemical studies. Neurosci Res. 2002;43(4):373-9. 
https://doi.org/10.1016/s0168-0102(02)00063-9

PMid: 12135780

12. Moussa AR, Ibraheem EM, Elboraey AN. Evaluation of changes in brain activity and cognitive function of diabetic patients wearing removable partial dentures. J Arab Soc Med Res. 2020;15(2):42-7. https://doi.org/10.4103/jasmr.jasmr_29_20

13. Toshio H, Masakazu M, Naoyuki S, Yoshikazu Y. Influence of denture treatment on brain function activity. Jpn Dent Sci Rev. 2011;47(1):56-66.

14. Banu RF, Veeravalli PT, Kumar VA. Comparative evaluation of changes in brain activity and cognitive function of edentulous patients, with dentures and two-implant supported mandibular overdenture-pilot study. Clin implant Dent Relat Res. 2016;18(3):580-7. https://doi.org/10.1111/cid.12336 PMid:25825258

15. Matsuda R, Yoneyama $\mathrm{Y}$, Morokuma M, Ohkubo C. Influence of vertical dimension of occlusion changes on the electroencephalograms of complete denture wearers. J Prosthodont Res. 2014;58(2):121-6. https://doi.org/10.1016/j. jpor.2014.01.003

PMid:24745815

16. Ozdemir K, Ozdemir HD, Polat NT, Turgut M, Sezer H. The effect of personality type on denture satisfaction. Int J Prosthodont. 2006;19(4):364-70. PMid: 16900819

17. Sulaiman $\mathrm{N}$, Hamid NH, Murat ZH, Taib MN. Initial Investigation of Human Physical Stress Level using Brainwaves. United States: IEEE Student Conference Research and Development; 2009. p. 230-3. https://doi.org/10.1109/scored.2009.5443088

18. Wheeler RE, Davidson RJ, Tomarken AJ. Frontal brain asymmetry and emotional reactivity: A biological substrate of affective style. Psychophysiology. 1993;30(1):82-9. https://doi. org/10.1111/j.1469-8986.1993.tb03207.x

PMid:8416065

19. Niemiec A, Lithgow B. Alpha-band characteristics in EEG spectrum indicate reliability of frontal brain asymmetry measures in diagnosis of depression. Conf Proc IEEE Eng Med Biol Soc. 2005;2005:7517-20. https://doi.org/10.1109/iembs.2005.1616251
PMid: 17282020

20. Morokuma M, Yoneyama Y, Matsuda R, Hosoi T, Ohkubo C. Influence of occlusal force on electroencephalograms in edentulous patients. J Prosthodont. 2015;24(7):532-7. https:// doi.org/10.1111/jopr.12263

PMid:25594138

21. Goiato MC, Do Prado Ribeiro P, Garcia AR, dos Santos DM. Complete denture masticatory efficiency: A literature review. J Calif Dent Assoc. 2018;36(9):683-6.

PMid: 18856170

22. Manns A, Miralles R, Palazzi C. EMG, bite force, and elongation of the masseter muscle under isometric voluntary contractions and variations of vertical dimension. J Prosthet Dent. 1979;42(6):67482. https://doi.org/10.1016/0022-3913(79)90200-2

PMid:292779

23. Abdel-Salam EB, Almahdy YF, Baraka YA, Mostafa HI. Effect of occlusal vertical dimension of complete denture on maximum biting force. Egypt Dent J. 2016;62(1):595-600. https://doi. org/10.21608/edj.2016.95073

24. George AZ. Biomechanics of edentulous state. In: Prosthodontic Treatment for Edentulous Patient. Complete Dentures and Implant-Supported Prostheses. $12^{\text {th }}$ ed. Philadelphia, PA: Elsevier; 2004. p. 483.

25. Abduo J. Safety of increasing vertical dimension of occlusion: A systematic review. Quintessence Int. 2012;43(5):369-80. PMid:22536588

26. Hirano $\mathrm{Y}$, Obata $\mathrm{T}$, Takahashi H, Tachibana A, Kuroiwa $\mathrm{D}$, Takahashi $\mathrm{T}$, et al. Effects of chewing on cognitive processing speed. Brain Cogn. 2013;81(3):376-81. https://doi.org/10.1016/j. bandc.2012.12.002

PMid:23375117

27. Perumal P, Chander GN, Anitha KV, Reddy JR, Muthukumar B. Power spectrum density analysis for the influence of complete denture on the brain function of edentulous patients-pilot study. J Adv Prosthodont. 2016;8(3):187-93. https://doi.org/10.4047/ jap.2016.8.3.187

PMid:27350852 